\title{
Islanding detection method using ridgelet probabilistic neural network in distributed generation
}

\begin{abstract}
One of the challenging issues for a grid-connected embedded generation is to find a suitable technique to detect an islanding problem. The technique must be able to differentiate islanding from other grid disturbances and disconnect distributed generation (DG) rapidly to prevent from safety hazards, power quality issues, equipment damage, as well as voltage and frequency instability. This study proposes a Slantlet transform as a signal processing method to extract the essential features to distinguish islanding from other disturbances. A ridgelet probabilistic neural network (RPNN) is utilized to classify islanding and grid disturbances. A modified differential evolution (MDF) algorithm with a new mutation phase, crossover process, and selection mechanism is proposed to train the RPNN. The results of the proposed technique show its capability and robustness to differentiate between islanding events and other grid disturbances
\end{abstract}

Keyword: Distributed generation; Islanding detection; Ridgelet probabilistic neural network; Discrete wavelet transform; Slantlet transform; Differential evolution 